\title{
Qualidade de vida dos surdos que se comunicam pela língua de sinais: revisão integrativa
}

Neuma Chaveiro(a)

Soraya Bianca Reis Duarte(b)

Adriana Ribeiro de Freitas ${ }^{(c)}$

Maria Alves Barbosa ${ }^{(d)}$

Celmo Celeno Porto(e)

Marcelo Pio de Almeida Fleck ${ }^{(f)}$

Chaveiro N, Duarte SBR, Freitas AR, Barbosa MA, Porto CC, Fleck MPA. Quality of life of deaf people who communicate in sign language: integrative review. Interface

(Botucatu). 2014; 18(48):101-14.

The purpose of this study was to review the scientific production on the healthrelated quality of life (HRQOL) of deaf people. This was an integrative review carried out in the Virtual Health Library, PubMed and CAPES (Coordination Office for Improvement of Higher Education Personnel) periodicals portal. The results indicated that anxiety and depression symptoms are greater among deaf people and may be related to difficulties in communication. People who experience communication problems avoid new social relationships and this may increase social isolation and diminish HRQOL. For deaf people who communicate in sign language, HRQOL can only be effectively evaluated by instruments that have been translated and adapted to their language. In conclusion, deafness has a negative impact on these individuals' HRQOL.

Keywords: Deafness. Sign language. Quality of life. Review.
O estudo objetivou revisar a produção científica sobre a qualidade de vida relacionada à saúde (QVRS) de surdos. Trata-se de uma revisão integrativa, realizada na Biblioteca Virtual em Saúde, PubMed e Portal de periódicos da Coordenação de Aperfeiçoamento de Pessoal de Nível Superior (Capes). Os resultados indicam que sintomas de ansiedade e depressão são mais acentuados nos surdos e podem estar relacionados a dificuldades de comunicação. As pessoas que vivenciam problemas de comunicação evitam novas relações sociais, e isso pode aumentar o isolamento social e reduzir a QVRS. Para os surdos que se comunicam pela Língua de Sinais, a QVRS só pode ser efetivamente avaliada por instrumentos traduzidos e adaptados em sua língua.

Conclui-se que a surdez tem um impacto negativo sobre a qualidade de vida relacionada à saúde (QVRS) de pessoas surdas.

Palavras-chave: Surdez. Linguagem de sinais. Qualidade de vida. Revisão.

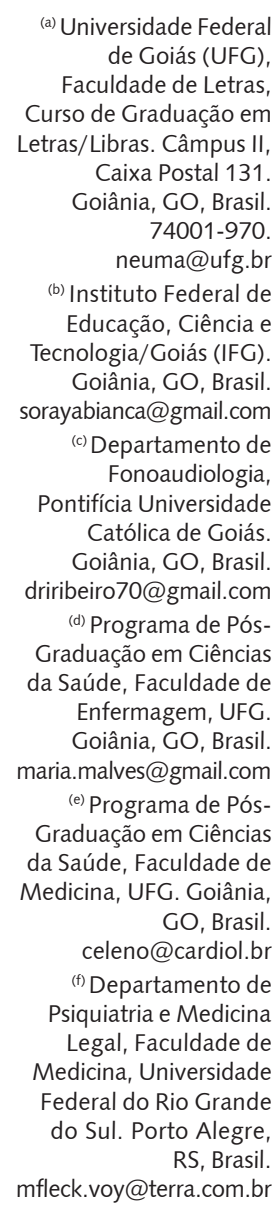




\section{Introdução}

Nas últimas décadas, o conceito de surdez passou por transformações históricas e culturais. O surdo deixa de ser considerado "deficiente" e passa a ser "diferente"; a surdez não é concebida como uma deficiência a ser curada, eliminada ou normalizada, e sim como uma diferença a ser respeitada. Nesse contexto, a pessoa surda pertence a uma comunidade minoritária, que partilha uma língua de sinais, valores culturais, hábitos e modos de socialização próprios¹.

Um fato a ser ressaltado é o de que a surdez distingue-se de outras deficiências, não pela deficiência física propriamente dita, mas pela dificuldade de estabelecer comunicação entre pessoas: os problemas de comunicação, no cotidiano dos surdos, são uma condição permanente, que acarreta graves consequências no seu desenvolvimento social, emocional e cognitivo².

As línguas de sinais são consideradas como língua, oficialmente, em vários países. A linguística lhes atribui o conceito de língua natural, com estruturas gramaticais próprias. Legalmente, vários países a reconhecem como meio de comunicação e expressão dos surdos ${ }^{3}$. No Brasil, a Língua Brasileira de Sinais (LIBRAS) foi reconhecida pela Lei Federal $n^{\circ} 10.436 / 02^{4}$.

Contrariamente a uma ideia preconcebida, não existe uma língua de sinais utilizada e compreendida universalmente. As línguas sinalizadas diferem-se umas das outras. Dessa forma, quando um surdo aprende uma segunda língua de sinais, ele utiliza sinais com sotaque estrangeiro. Há vários contrastes entre as línguas orais e as línguas de sinais; o que é reconhecido por palavra nas línguas orais, denomina-se sinal nas línguas de sinais. A modalidade de produção é outro contraste; as línguas de sinais são espaço-visuais, pois o sistema de signos compartilhado é recebido pelos olhos e sua produção realizada pelas mãos no espaço, ao passo que as línguas orais são oral-auditivas ${ }^{1,5}$.

A história social da surdez retrata quanto o surdo e, mais recentemente sua comunidade, têm sido submetidos ao controle dos profissionais da saúde e da sociedade ouvinte, exatamente por desafiarem os limites normativos do normal e do patológico. Para a comunidade surda, o problema de comunicação dos surdos não é de origem orgânica, e sim social e cultural. Seu argumento é o de que os surdos são considerados deficientes pelo simples fato de não usarem a mesma língua da comunidade majoritária, no caso a língua oral-auditiva ${ }^{1,3,6}$.

$\mathrm{Na}$ área da saúde, a surdez precisa ser vista como uma diferença a ser politicamente reconhecida e como uma experiência visual. A comunidade surda, nessa concepção, deixa de ser considerada apenas pelo déficit auditivo e passa a ser respeitada pela sua identidade, com valores culturais. Nessa perspectiva, a posição das pessoas surdas passa a ser definida em termos culturais e linguísticos ${ }^{5}$.

A surdez pode reduzir a qualidade de vida e se qualificar como um distúrbio crônico. A probabilidade de os surdos sofrerem alterações psicológicas é de três a cinco vezes maior que a das pessoas ouvintes. Uma possível explicação é a de que uma perda auditiva antes dos três anos de idade pode prejudicar a aquisição da língua oral, que é o meio de comunicação no ambiente familiar?.

Vale ressaltar que a maioria dos surdos $(91,7 \%)$ tem pais ouvintes e sem experiência para lidar com pessoas surdas. Assim, eles vivenciam o isolamento, por fazerem parte de famílias ouvintes, que não dominam a língua de sinais. Crescer com dificuldades de comunicação pode comprometer o desempenho das habilidades sociais e emocionais, e ainda acarretar uma qualidade de vida inferior ${ }^{8}$.

Transtornos psicológicos em decorrência da perda auditiva podem gerar impacto negativo nas dimensões psicossociais dos surdos. No entanto, a surdez tem sido investigada, na área da saúde, sobretudo numa perspectiva clínico-terapêutica ou oralista, ficando a desejar pesquisas sobre a Qualidade de Vida Relacionada à Saúde (QVRS) das pessoas surdas que utilizam a língua de sinais. Assim, sem um desfecho válido e confiável em língua de sinais, para investigar a QVRS dos surdos, a eficácia das intervenções de saúde para essa população pode estar comprometida.

Considerando que existem medidas de QVRS, inclusive com instrumentos padronizados e recomendados para mensurá-las em diversas culturas, traduzidos e validados em diferentes idiomas, por que esse procedimento não é aplicado à população surda que usa a língua de sinais? Como avaliar a qualidade de vida dos surdos com instrumentos em uma língua que eles não dominam? Para uma avaliação fidedigna da QVRS das pessoas surdas, é de suma importância que se considerem os aspectos linguísticos e culturais das pessoas surdas. 


\section{Objetivos}

Revisar a produção científica sobre QVRS de surdos que se comunicam pela língua de sinais e verificar se os instrumentos de avaliação de qualidade de vida, utilizados nos estudos, foram traduzidos para língua de sinais.

\section{Procedimentos metodológicos}

(g) Texto elaborado com base em tese de doutorado de Neuma Chaveiro, que contou com dois financiamentos: 1 Projeto de Pesquisa: Qualidade de vida relacionada à saúde das pessoas surdas que usam a Língua Brasileira de Sinais e a assistência oferecida pelo Sistema Único de Saúde, do DECIT/SCTIE/MS, por intermédio do Conselho Nacional de Pesquisa e Desenvolvimento Tecnológico (CNPq), e o apoio da Fundação de Amparo à Pesquisa do Estado de Goiás (FAPEG), processon. 200910267000540. 2 Bolsa de formação de Doutorado, agência financiadora: FAPEG processo $\mathrm{n}$.

200910267000539.
Trata-se de uma revisão integrativa da literatura(g) ${ }^{(g)}$ método de análise de pesquisas em que conclusões de estudos anteriores são sintetizadas, a fim de que se formulem inferências sobre um tópico específico, com a finalidade de contribuir para o conhecimento do tema investigado?.

O processo de elaboração deste artigo seguiu as etapas propostas pela Revisão Integrativa da Literatura, quais sejam: identificação do tema; elaboração da pergunta norteadora; estabelecimento de critérios para inclusão e exclusão de estudos/ busca na literatura; definição das características dos estudos; resultados; e apresentação da revisão integrativa ${ }^{9}$.

A referida revisão teve as seguintes questões norteadoras: como têm sido realizadas as pesquisas sobre a QVRS de pessoas surdas que se comunicam pela língua de sinais? Os aspectos linguísticos e culturais têm sido considerados nesses estudos? Existem instrumentos de avaliação de qualidade de vida traduzidos para língua de sinais?

\section{Busca na literatura}

A revisão da literatura iniciou-se em maio de 2011, com término em abril de 2013; foi realizado o levantamento da produção científica na Biblioteca Virtual em Saúde (BVS), na busca integrada com as palavras-chave: mental, health, deaf; foram encontrados 151 artigos. Pelo nome do autor, foi encontrado um artigo. $\mathrm{Na}$ base de dados PubMed (National Library of Medicine), foram localizados 157 artigos com as palavras-chaves quality, life e deaf. Os artigos encontrados estavam na BVS, indexados nos bancos de dados da Medline (Medical Literature Analysis and Retrieval Sistem on-line), PubMed, Lilacs (Literatura Latino-Americana de Ciências da Saúde), IBECS (Índice Bibliográfico Espanhol em Ciências da Saúde), MedCarib (Literatura do Caribe em Ciências) e Cochrane Library. Foi encontrado, também, um artigo indexado no portal de periódicos da Coordenação de Aperfeiçoamento de Pessoal de Nível Superior (Capes).

O Quadro 1 apresenta a amostra obtida na BVS/Medline, PubMed e Capes, de acordo com o método de busca e com as palavras-chave.

Os artigos foram selecionados inicialmente com a leitura do título e do resumo, a fim de verificar a sua adequação com a questão norteadora da presente investigação; quando adequados, foram lidos e analisados na íntegra.

Os critérios de inclusão que compuseram a amostra foram: artigos que apresentaram relação entre qualidade de vida e pessoas surdas que se comunicam pela língua de sinais, artigos científicos indexados nas bases de dados da BVS, PubMed e publicados entre o período de 2000 a 2012, nos idiomas português, espanhol e inglês; já os critérios de exclusão foram: artigos com o objetivo de avaliar a qualidade de vida relacionada à reabilitação oral e à eficácia do implante coclear ou a aparelhos auditivos na oralização, desconsiderando-se a língua de sinais como meio de comunicação e artigos em idiomas diferentes do português, espanhol e inglês. 
Quadro 1. Amostra obtida na BVS, PubMed e Capes

\begin{tabular}{|c|c|c|c|}
\hline $\begin{array}{l}\text { Base de dados } \\
\text { consultada }\end{array}$ & $\begin{array}{l}\text { Método de } \\
\text { busca }\end{array}$ & Palavras-chave & Artigos \\
\hline $\begin{array}{l}\text { Lilacs, Medline, } \\
\text { PubMed, IBECS, } \\
\text { MedCarib,Cochrane } \\
\text { Library }\end{array}$ & Integrado & Mental, health, deaf & $\begin{array}{l}\text { Total de artigos encontrados: } 151 \\
\text { Total de artigos rejeitados pelo título: } 90 \\
\text { Total de artigos rejeitados pelo resumo: } 52 \\
\text { Total de artigos rejeitados pela leitura integral: } 3 \\
\text { Total de artigos incluídos na revisão da literatura: } 6\end{array}$ \\
\hline Medline & Autor & - & $\begin{array}{l}\text { Total de artigos encontrados e incluídos na revisão da } \\
\text { literatura: } 1\end{array}$ \\
\hline PubMed & & Quality, life, deaf & $\begin{array}{l}\text { Total de artigos encontrados: } 157 \\
\text { Total de artigos rejeitados pelo título: } 133 \\
\text { Total de artigos rejeitados pelo resumo: } 13 \\
\text { Total de artigos rejeitados pela leitura integral: } 5 \\
\text { Total de artigos incluídos na revisão da literatura: } 6\end{array}$ \\
\hline Periódicos da Capes & $\begin{array}{l}\text { Título do } \\
\text { Periódico e } \\
\text { do artigo }\end{array}$ & - & Total de artigos encontrados: 1 \\
\hline
\end{tabular}

\section{Definição das características dos estudos}

Dos artigos excluídos, um número expressivo tinha como objetivo pesquisar a qualidade de vida relacionada ao implante coclear ou ao uso de aparelhos auditivos. Dos 157 artigos encontrados na base de dados da PubMed, setenta tratavam da influência do implante coclear na qualidade de vida dos Deficientes Auditivos (DA), pois, frequentemente, as pessoas com implante coclear ou que usam aparelhos auditivos fazem a opção pela filosofia oralista em sua educação, portanto são DA oralizados e não usam a língua de sinais como meio de comunicação; assim, esses artigos não estavam em consonância com o objetivo deste estudo.

Em relação ao idioma, a maior parte foi publicada em inglês. Dos 151 artigos encontrados na BVS, nas bases de dados Lilacs, Medline, PubMed, IBECS, MedCarib, Cochrane Library, método de busca integrado, 138 eram em inglês, cinco em português, dois em alemão, dois em espanhol, dois em francês, um em sérvio e um em turco.

Nessa mesma busca, em relação ao ano de publicação, 38 artigos selecionados datavam de 1971 a 1997. Apesar de, no presente estudo, ter-se definido que as publicações científicas avaliadas estariam concentradas a partir do ano 2000, foi feita a leitura dos títulos e resumos dos artigos anteriores a 2000. Verificou-se que não correspondiam às questões norteadoras dessa revisão de literatura. Deve-se salientar que a maior produtividade encontra-se, sobretudo, nos últimos cinco anos.

\section{Resultados}

Para análise e interpretação dos 14 artigos científicos selecionados, foi feito um quadro sinóptico, que apreciou os seguintes aspectos: periódico, país de realização do estudo/ país de publicação/idioma de publicação, títulos, autoria, instrumento utilizado, objetivos, resultados e conclusão.

No Quadro 2 encontra-se a síntese de três artigos científicos que investigaram a qualidade de vida com instrumentos traduzidos para língua de sinais. 
Quadro 2. Artigos com instrumentos traduzidos para língua de sinais

\begin{tabular}{|c|c|c|c|c|c|}
\hline $\begin{array}{l}\text { Periódico/país } \\
\text { de realização/ } \\
\text { país de } \\
\text { publicação/ } \\
\text { Idioma } \\
\text { publicado/Ano }\end{array}$ & Título & Instrumento & Objetivo & Resultado & Conclusão \\
\hline $\begin{array}{l}\text { Journal of Deaf } \\
\text { Studies and Deaf } \\
\text { Education/ } \\
\text { Noruega/ } \\
\text { Estados Unidos/ } \\
\text { Inglês/ } 2007\end{array}$ & $\begin{array}{l}\text { Mental } \\
\text { health in } \\
\text { deaf adults: } \\
\text { symptoms of } \\
\text { anxiety and } \\
\text { depression } \\
\text { among } \\
\text { hearing and } \\
\text { deaf } \\
\text { individuals }\end{array}$ & $\begin{array}{l}\text { Versão abreviada } \\
\text { do Hopkins } \\
\text { Symptom } \\
\text { Checklist. }\end{array}$ & $\begin{array}{l}\text { Analisar a saúde } \\
\text { mental de surdos em } \\
\text { comparação com a } \\
\text { de ouvintes. }\end{array}$ & $\begin{array}{l}\text { Os surdos são mais } \\
\text { vulneráveis aos } \\
\text { problemas de saúde } \\
\text { mental. A comparação } \\
\text { entre surdos e ouvintes } \\
\text { mostrou que os sintomas } \\
\text { de ansiedade e de } \\
\text { depressão são maiores } \\
\text { nos grupo de surdos. }\end{array}$ & $\begin{array}{l}\text { É preciso concentrar } \\
\text { mais atenção na saúde } \\
\text { mental dos surdos. A } \\
\text { sociedade deve estar } \\
\text { ciente dos problemas } \\
\text { de saúde mental a que } \\
\text { os surdos estão sujeitos. }\end{array}$ \\
\hline $\begin{array}{l}\text { Acta Psychiatrica } \\
\text { Scandinavica/ } \\
\text { Áustria e } \\
\text { Alemanha/ } \\
\text { Dinamarca / } \\
\text { Inglês/ } 2007\end{array}$ & $\begin{array}{l}\text { Mental } \\
\text { distress and } \\
\text { quality of life } \\
\text { in the hard } \\
\text { of hearing }\end{array}$ & $\begin{array}{l}\text { WHOQOL-Brief, } \\
\text { General Health } \\
\text { Questionnaire } \\
\text { GHQ-12 e o Brief } \\
\text { Symptom } \\
\text { Inventory. }\end{array}$ & $\begin{array}{l}\text { Comparar os níveis } \\
\text { de estresse } \\
\text { psicológico e a QV } \\
\text { entre DA, surdos } \\
\text { usuários da LS e a } \\
\text { população ouvinte. }\end{array}$ & $\begin{array}{l}\text { Os DA têm as piores } \\
\text { relações sociais, se } \\
\text { comparados com os } \\
\text { surdos que usam a LS e } \\
\text { com os ouvintes. A QV } \\
\text { dos DA está relacionada } \\
\text { ao desempenho do } \\
\text { aparelho auditivo. }\end{array}$ & $\begin{array}{l}\text { Psiquiatras precisam } \\
\text { estar cientes de que } \\
\text { pacientes DA podem ter } \\
\text { um maior isolamento do } \\
\text { que as pessoas surdas } \\
\text { que usam a LS. }\end{array}$ \\
\hline $\begin{array}{l}\text { Social Psychiatry } \\
\text { and Psychiatric } \\
\text { Epidemiology/ } \\
\text { Áustria e } \\
\text { Alemanha / } \\
\text { Alemanha/ } \\
\text { Inglês/ 2005 }\end{array}$ & $\begin{array}{l}\text { Mental } \\
\text { distress and } \\
\text { quality of life } \\
\text { in a deaf } \\
\text { population }\end{array}$ & $\begin{array}{l}\text { WHOQOL-Brief, } \\
\text { General Health } \\
\text { Questionnaire - } \\
12 \text { e o Brief } \\
\text { Symptom } \\
\text { Inventory. }\end{array}$ & $\begin{array}{l}\text { Avaliar transtornos } \\
\text { mentais e a QV de } \\
\text { surdos da Áustria. }\end{array}$ & $\begin{array}{l}\text { Os surdos têm uma QV } \\
\text { pior do que os ouvintes } \\
\text { nos domínios físico e } \\
\text { psicológico. No domínio } \\
\text { das relações sociais, não } \\
\text { houve diferença } \\
\text { significativa. Os } \\
\text { resultados com o GHQ- } \\
12 \text { e do BSI mostram } \\
\text { níveis mais elevados de } \\
\text { problemas emocionais } \\
\text { entre os surdos. }\end{array}$ & $\begin{array}{l}\text { Apesar da QV inferior e } \\
\text { de um maior nível de } \\
\text { estresse mental, a } \\
\text { semelhança com a } \\
\text { população ouvinte, no } \\
\text { domínio das relações } \\
\text { sociais, mostra que nem } \\
\text { todos os domínios estão } \\
\text { afetados. Os resultados } \\
\text { indicam a necessidade } \\
\text { de serviços de saúde } \\
\text { acessíveis aos surdos, } \\
\text { com uso de sua língua. }\end{array}$ \\
\hline
\end{tabular}

O Quadro 3 contém seis artigos que investigaram a qualidade de vida com instrumentos sem tradução ou com tradução face a face/tradução simultânea para língua de sinais.

No Quadro 4 estão cinco artigos que descrevem a metodologia de tradução dos instrumentos para língua de sinais.

\section{Apresentação da revisão integrativa}

Para efeitos da discussão dessa revisão integrativa, será seguida a ordem de apresentação dos resultados. Primeiro, estarão em discussão os artigos científicos que investigaram a qualidade de vida com instrumentos traduzidos para a língua de sinais (Quadro 2); depois, os artigos científicos que investigaram a qualidade de vida com instrumentos sem tradução ou com tradução face a face/ tradução simultânea para a língua de sinais (Quadro 3); por último, os artigos que descreveram a tradução de instrumentos para a língua de sinais (Quadro 4). 
QUALIDADE DE VIDA DOS SURDOS $\ldots$

Quadro 3. Artigos sem tradução ou com tradução simultânea para língua de sinais

\begin{tabular}{|c|c|c|c|c|c|}
\hline $\begin{array}{c}\text { Periódico/País } \\
\text { de realização/ } \\
\text { País de } \\
\text { publicação/ } \\
\text { Idioma } \\
\text { publicado/Ano }\end{array}$ & Título & Instrumento & Objetivo & Resultado & Conclusão \\
\hline $\begin{array}{l}\text { European Child } \\
\& \text { Adolescent } \\
\text { Psychiatry/ } \\
\text { Áustria/ } \\
\text { Alemanha/ } \\
\text { Inglês/ } 2008\end{array}$ & $\begin{array}{l}\text { Mental health } \\
\text { and quality of } \\
\text { life in deaf } \\
\text { pupils }\end{array}$ & $\begin{array}{l}\text { Strengths and } \\
\text { Difficulties } \\
\text { Questionnaire } \\
\text { (SDQ) Inventory } \\
\text { the Quality of } \\
\text { Life in Children } \\
\text { and adolescents. }\end{array}$ & $\begin{array}{l}\text { Avaliar os aspectos } \\
\text { de saúde mental e } \\
\text { a QV em uma } \\
\text { amostra de alunos } \\
\text { surdos. }\end{array}$ & $\begin{array}{l}\text { Crianças surdas } \\
\text { pontuaram mais no } \\
\text { SDQ do que as } \\
\text { ouvintes. As diferenças } \\
\text { foram mais acentuadas } \\
\text { nos problemas de } \\
\text { conduta, emocionais e } \\
\text { de relacionamento, e } \\
\text { menos para } \\
\text { hiperatividade. }\end{array}$ & $\begin{array}{l}\text { É necessário apoio para } \\
\text { crianças surdas com } \\
\text { relação à saúde mental } \\
\text { e QV, independente do } \\
\text { grau de perda auditiva. } \\
\text { Serviços de saúde } \\
\text { mental para crianças } \\
\text { surdas e DA devem ser } \\
\text { incluídos em ambiente } \\
\text { educacional. }\end{array}$ \\
\hline $\begin{array}{l}\text { Psychosomatic } \\
\text { Medicin / } \\
\text { Holanda/ } \\
\text { Estados Unidos/ } \\
\text { Inglês/ } 2002\end{array}$ & $\begin{array}{l}\text { Determinants } \\
\text { of mental } \\
\text { distress in } \\
\text { adults with a } \\
\text { severe } \\
\text { auditory } \\
\text { impairment: } \\
\text { differences } \\
\text { between } \\
\text { prelingual and } \\
\text { postlingual } \\
\text { deafness }\end{array}$ & $\begin{array}{l}\text { General Health } \\
\text { Questionnaire } \\
\text { General; } \\
\text { Symptom } \\
\text { Checklist } \\
\text { (SCL-8D). }\end{array}$ & $\begin{array}{l}\text { Investigar os } \\
\text { determinantes de } \\
\text { saúde mental entre } \\
\text { DA com perda } \\
\text { auditiva severa, } \\
\text { separados pela } \\
\text { idade do início da } \\
\text { perda auditiva. }\end{array}$ & $\begin{array}{l}\text { Nos surdos, o } \\
\text { sofrimento mental } \\
\text { apresentou taxas mais } \\
\text { elevadas do que na } \\
\text { população em geral. } \\
\text { Nas duas categorias, o } \\
\text { risco de sofrimento } \\
\text { mental foi maior entre } \\
\text { aqueles com mais } \\
\text { problemas de } \\
\text { comunicação, baixos } \\
\text { níveis de autoestima e } \\
\text { uma menor aceitação } \\
\text { da perda auditiva. }\end{array}$ & $\begin{array}{l}\text { O nível de saúde } \\
\text { mental difere entre os } \\
\text { DA e a população } \\
\text { ouvinte, mas não tanto } \\
\text { como, às vezes, é } \\
\text { sugerido. A doença } \\
\text { mental é maior em } \\
\text { certas categorias de } \\
\text { DA. }\end{array}$ \\
\hline $\begin{array}{l}\text { Journal of Deaf } \\
\text { Studies and } \\
\text { Deaf Education/ } \\
\text { Alemanha/ } \\
\text { Estados Unidos/ } \\
\text { Inglês/ } 2008\end{array}$ & $\begin{array}{l}\text { Self-esteem } \\
\text { and } \\
\text { satisfaction } \\
\text { with life of } \\
\text { deaf and hard- } \\
\text { of-hearing } \\
\text { people-a } \\
\text { resource- } \\
\text { oriented } \\
\text { approach to } \\
\text { identity work }\end{array}$ & $\begin{array}{l}\text { Questionário } \\
\text { elaborado pelos } \\
\text { pesquisadores. }\end{array}$ & $\begin{array}{l}\text { Verificar as inter- } \\
\text { relações entre } \\
\text { cultura, aspectos } \\
\text { psicológicos, } \\
\text { autoestima e a } \\
\text { satisfação com a } \\
\text { vida dos surdos e } \\
\text { DA. }\end{array}$ & $\begin{array}{l}\text { A disponibilidade de } \\
\text { recursos psicológicos é } \\
\text { importante para a } \\
\text { qualidade da } \\
\text { autoestima e a } \\
\text { satisfação com a vida. } \\
\text { Por outro lado, o } \\
\text { bem-estar psicológico } \\
\text { está associado a uma } \\
\text { boa condição } \\
\text { comunicativa, bem } \\
\text { como ao nível de } \\
\text { educação. }\end{array}$ & $\begin{array}{l}\text { A missão dos } \\
\text { educadores parece ser } \\
\text { oportunizar boas } \\
\text { condições de } \\
\text { comunicação para } \\
\text { crianças surdas ou com } \\
\text { DA, e otimizar o } \\
\text { desempenho } \\
\text { acadêmico. Dessa } \\
\text { forma, uma boa base } \\
\text { pode ser colocada para } \\
\text { O desenvolvimento da } \\
\text { QV. }\end{array}$ \\
\hline
\end{tabular}

continua

\section{Artigos que investigaram a qualidade de vida com instrumentos traduzidos para a língua de sinais}

A investigação para essa revisão de literatura revelou que muitas pesquisas são desenvolvidas com o intuito de mensurar a QVRS em diferentes populações. A avaliação descrita, pelos pacientes, sobre a sua qualidade de vida pode determinar se os tratamentos estão atingindo os objetivos propostos. Esses desfechos podem ser denominados de indicadores de qualidade de vida ${ }^{10}$. No entanto, poucos estudos mostram a QVRS da população surda que utiliza língua de sinais. Somente nove artigos investigaram a qualidade de vida das pessoas surdas que se comunicam pela língua de sinais e apenas três destes 
Quadro 3. continuação

\begin{tabular}{|c|c|c|c|c|c|}
\hline $\begin{array}{c}\text { Periódico/País } \\
\text { de realização/ } \\
\text { País de } \\
\text { publicação/ } \\
\text { Idioma } \\
\text { publicado/Ano }\end{array}$ & Título & Instrumento & Objetivo & Resultado & Conclusão \\
\hline $\begin{array}{l}\text { Archives of } \\
\text { Gerontology } \\
\text { and Geriatrics/ } \\
\text { Suécia/ Irlanda/ } \\
\text { Inglês/ } 2003\end{array}$ & $\begin{array}{l}\text { Aspects of } \\
\text { quality of life } \\
\text { in persons } \\
\text { with pre- } \\
\text { lingual } \\
\text { deafness } \\
\text { using sign } \\
\text { language: } \\
\text { subjective } \\
\text { wellbeing, ill- } \\
\text { health } \\
\text { symptoms, } \\
\text { depression } \\
\text { and insomnia }\end{array}$ & $\begin{array}{l}\text { Gothenburg } \\
\text { Quality of Life } \\
\text { (GQL), Geriatric } \\
\text { Depression } \\
\text { Scale (GDS), } \\
\text { Livingston's } \\
\text { Sleep Scale. }\end{array}$ & $\begin{array}{l}\text { Investigar os } \\
\text { aspectos da QV } \\
\text { de pessoas surdas } \\
\text { pré-linguais, } \\
\text { usuárias da língua } \\
\text { de sinais, } \\
\text { expressos pelo } \\
\text { bem-estar, por } \\
\text { sintomas de } \\
\text { saúde-doença, } \\
\text { por depressão e } \\
\text { por insônia. }\end{array}$ & $\begin{array}{l}\text { Um terço dos surdos } \\
\text { demonstraram sintomas } \\
\text { depressivos e cerca de } \\
\text { dois terços, insônia. } \\
\text { Houve correlação } \\
\text { significativa entre a } \\
\text { insônia, os sintomas } \\
\text { depressivos e um menor } \\
\text { bem-estar. Os resultados } \\
\text { reforçaram a hipótese de } \\
\text { que os sintomas } \\
\text { depressivos e os } \\
\text { distúrbios do sono são } \\
\text { mais frequentes entre os } \\
\text { idosos surdos usuários da } \\
\text { LS do que entre } \\
\text { ouvintes. }\end{array}$ & $\begin{array}{l}\text { Apesar de os resultados } \\
\text { apresentados serem } \\
\text { únicos e contribuirem } \\
\text { para o aumento do } \\
\text { nível de QV da } \\
\text { população de idosos } \\
\text { surdos, falta } \\
\text { pesquisadores com } \\
\text { habilidades em LS. }\end{array}$ \\
\hline $\begin{array}{l}\text { Journal of Deaf } \\
\text { Studies and } \\
\text { Deaf Education/ } \\
\text { Alemanha/ } \\
\text { Estados Unidos/ } \\
\text { Inglês/ } 2010\end{array}$ & $\begin{array}{l}\text { Health- } \\
\text { Related } \\
\text { Quality of } \\
\text { Life and } \\
\text { Classroom } \\
\text { Participation } \\
\text { of Deaf and } \\
\text { Hard-of- } \\
\text { Hearing } \\
\text { Students in } \\
\text { General } \\
\text { Schools }\end{array}$ & $\begin{array}{l}\text { Inventory of } \\
\text { Life Quality of } \\
\text { Children and } \\
\text { Youth (ILC) e } \\
\text { um questionario } \\
\text { de participação } \\
\text { em sala de aula. }\end{array}$ & $\begin{array}{l}\text { Avaliar a QVRS } \\
\text { de estudantes } \\
\text { Surdos/DA, em } \\
\text { sala inclusiva. } \\
\text { Verificar se o ILC } \\
\text { para crianças } \\
\text { ouvintes pode } \\
\text { ser usado para } \\
\text { avaliar a QVRS de } \\
\text { Surdos/DA. } \\
\text { Examinar a } \\
\text { correlação entre } \\
\text { QV e } \\
\text { participação em } \\
\text { sala de aula. }\end{array}$ & $\begin{array}{l}\text { As correlações } \\
\text { mostraram que os } \\
\text { domínios das atividades } \\
\text { escolares e sociais foram } \\
\text { mais importantes para a } \\
\text { QVRS dos estudantes } \\
\text { surdos e DA do que dos } \\
\text { alunos ouvintes. A QVRS } \\
\text { das duas amostras, } \\
\text { surdos e DA, obteve } \\
\text { escores mais elevados } \\
\text { para experiências } \\
\text { escolares, de saúde física } \\
\text { e mental e QV global, } \\
\text { embora a diferenças } \\
\text { sejam pequenas. }\end{array}$ & $\begin{array}{l}\text { A QVRS de alunos } \\
\text { surdos/DA em escolas } \\
\text { inclusivas não difere da } \\
\text { QVRS dos estudantes } \\
\text { ouvintes. Mas precisa- } \\
\text { se considerar: há uma } \\
\text { tendência para alunos } \\
\text { surdos/DA serem } \\
\text { menos satisfeitos com a } \\
\text { QV. }\end{array}$ \\
\hline $\begin{array}{l}\text { Scandinavian } \\
\text { Audiology/ } \\
\text { Suécia/Suécia/ } \\
\text { Inglês/2001 }\end{array}$ & $\begin{array}{l}\text { Severe- } \\
\text { profound } \\
\text { hearing } \\
\text { impairment } \\
\text { and health- } \\
\text { related } \\
\text { quality of life } \\
\text { among post- } \\
\text { lingual } \\
\text { deafened } \\
\text { Swedish } \\
\text { adults }\end{array}$ & $\begin{array}{l}\text { Nottingham } \\
\text { Health Profile } \\
\text { (NHP), } \\
\text { Questionário } \\
\text { sobre estado } \\
\text { biopsicossocial. }\end{array}$ & $\begin{array}{l}\text { Descrever a } \\
\text { relação } \\
\text { diagnóstico } \\
\text { audiológico e } \\
\text { avaliação da } \\
\text { QVRS de acordo } \\
\text { com o PHN; } \\
\text { obter medidas de } \\
\text { QVRS em relação } \\
\text { à idade das } \\
\text { pessoas com } \\
\text { perda auditiva } \\
\text { pós-lingual; } \\
\text { comparar a } \\
\text { QVRS com a } \\
\text { população em } \\
\text { geral. }\end{array}$ & $\begin{array}{l}\text { As pessoas com perda } \\
\text { auditiva profunda } \\
\text { relataram menor QVRS. } \\
\text { Diferenças significativas } \\
\text { foram obtidas por falta } \\
\text { de energia, reações } \\
\text { emocionais e isolamento } \\
\text { social. As mulheres } \\
\text { apresentaram QVRS } \\
\text { mais baixa que os } \\
\text { homens. Deficiência } \\
\text { auditiva severa-profunda } \\
\text { está associada a um } \\
\text { impacto sobre a QVRS, } \\
\text { especialmente nos } \\
\text { domínios emocionais e } \\
\text { sociais. }\end{array}$ & $\begin{array}{l}\text { DA profundos } \\
\text { constituem um grupo } \\
\text { de risco com pior } \\
\text { ajustamento } \\
\text { psicossocial e precisam } \\
\text { de maior atenção e } \\
\text { apoio. }\end{array}$ \\
\hline
\end{tabular}

QV (Qualidade de Vida); DA (Deficiente Auditivo); LS (Língua de Sinais); QVRS (Qualidade de Vida Relacionada à Saúde) 
QUALIDADE DE VIDA DOS SURDOS ...

Quadro 4. Artigos que descrevem a tradução de instrumentos para língua de sinais

\begin{tabular}{|c|c|c|c|c|c|}
\hline $\begin{array}{l}\text { Periódico/País } \\
\text { de realização/ } \\
\text { País de } \\
\text { publicação/ } \\
\text { Idioma/Ano }\end{array}$ & Título & Instrumento & Objetivo & Resultado & Conclusão \\
\hline $\begin{array}{l}\text { Australian and } \\
\text { New Zealand } \\
\text { Journal of } \\
\text { Psychiatry/ } \\
\text { Austrália / } \\
\text { Austrália / } \\
\text { Inglês / } 2009\end{array}$ & $\begin{array}{l}\text { Validation of } \\
\text { an Australian } \\
\text { sign language } \\
\text { instrument of } \\
\text { outcome } \\
\text { measurement } \\
\text { for adults in } \\
\text { mental health } \\
\text { settings }\end{array}$ & $\begin{array}{l}\text { Outcome Rating } \\
\text { Scale (ORS). } \\
\text { Versão } \\
\text { Australiana da } \\
\text { Depression } \\
\text { Anxiety Stress } \\
\text { Scale-21 } \\
\text { (DASS-21) }\end{array}$ & $\begin{array}{l}\text { Examinar a } \\
\text { confiabilidade, a } \\
\text { validade e a } \\
\text { aceitabilidade de } \\
\text { uma versão Língua } \\
\text { de Sinais } \\
\text { Australiana do ORS } \\
\text { (ORS-Auslan). }\end{array}$ & $\begin{array}{l}\text { A consistência interna } \\
\text { foi aceitável. A validade } \\
\text { de construto foi } \\
\text { estabelecida. A } \\
\text { aceitabilidade ficou } \\
\text { evidente na taxa de } \\
\text { conclusão de } 93 \% \text { em } \\
\text { comparação com } 63 \% \\
\text { para a DASS-21- } \\
\text { Australiana. }\end{array}$ & $\begin{array}{l}\text { O desfecho disponível } \\
\text { na versão em Língua de } \\
\text { Sinais Australiana pode } \\
\text { ser usado em uma } \\
\text { ampla variedade nas } \\
\text { avaliações de saúde. }\end{array}$ \\
\hline $\begin{array}{l}\text { Public Health } \\
\text { Nursing/ } \\
\text { Estados Unidos/ } \\
\text { Estados Unidos/ } \\
\text { Inglês/ } 2008\end{array}$ & $\begin{array}{l}\text { Translation of } \\
\text { the } \\
\text { multidimensional } \\
\text { health locus of } \\
\text { control scales } \\
\text { for users of } \\
\text { American sign } \\
\text { language }\end{array}$ & $\begin{array}{l}\text { Multidimensional } \\
\text { Health Locus of } \\
\text { Control (MHLC) } \\
\text { em ASL. }\end{array}$ & $\begin{array}{l}\text { Descrever a } \\
\text { tradução do } \\
\text { Multidimensional } \\
\text { Health Locus of } \\
\text { Control (MHLC) } \\
\text { em ASL. }\end{array}$ & $\begin{array}{l}\text { Identificaram } \\
\text { problemas culturais e } \\
\text { de linguagem; } 09 \text { dos } \\
24 \text { itens foram } \\
\text { diretamente } \\
\text { traduzíveis em ASL. } \\
\text { Nos demais itens, } \\
\text { foram necessárias } \\
\text { discussões para } \\
\text { alcançar equivalência } \\
\text { com a ASL. }\end{array}$ & $\begin{array}{l}\text { O MHLC/ ASL está } \\
\text { pronto para validação } \\
\text { no âmbito da } \\
\text { comunidade surda. }\end{array}$ \\
\hline $\begin{array}{l}\text { Nursing } \\
\text { Research/ } \\
\text { Estados Unidos/ } \\
\text { Estados Unidos/ } \\
\text { Inglês/ } 2006\end{array}$ & $\begin{array}{l}\text { Challenges in } \\
\text { language, } \\
\text { culture, and } \\
\text { modality: } \\
\text { translating } \\
\text { English } \\
\text { measures into } \\
\text { American sign } \\
\text { language }\end{array}$ & $\begin{array}{l}\text { Self-Rated } \\
\text { Abilities for } \\
\text { Health Practices } \\
\text { (SRAHP) e a } \\
\text { versão em ASL. }\end{array}$ & $\begin{array}{l}\text { Converter medidas } \\
\text { em LS e avaliar a } \\
\text { equivalência das } \\
\text { versões ASL para } \\
\text { medidas em } \\
\text { versões do Inglês. }\end{array}$ & $\begin{array}{l}\text { A média dos escores } \\
\text { da versão ASL foram } \\
\text { significativamente } \\
\text { menores para amostra } \\
\text { de surdos, embora a } \\
\text { consistência interna } \\
\text { permanecesse alta para } \\
\text { a nova versão em ASL. }\end{array}$ & $\begin{array}{l}\text { A abordagem é } \\
\text { apropriada para alterar } \\
\text { as formas escritas para } \\
\text { outras modalidades, } \\
\text { tais como a ASL. }\end{array}$ \\
\hline $\begin{array}{l}\text { Social Psychiatry } \\
\text { and Psychiatric } \\
\text { Epidemiology/ } \\
\text { Áustria/ } \\
\text { Alemanha/ } \\
\text { Inglês/ } 2005\end{array}$ & $\begin{array}{l}\text { An innovative } \\
\text { and reliable } \\
\text { way of } \\
\text { measuring } \\
\text { health-related } \\
\text { quality of life } \\
\text { and mental } \\
\text { distress in the } \\
\text { deaf } \\
\text { community }\end{array}$ & $\begin{array}{l}\text { WHOQOL-Brief, } \\
\text { General Health } \\
\text { Questionnaire } \\
\text { (GHQ-12) e o } \\
\text { Brief Symptom } \\
\text { Inventory (BSI). }\end{array}$ & $\begin{array}{l}\text { Descrever o } \\
\text { desenvolvimento } \\
\text { de um programa } \\
\text { de computador } \\
\text { para avaliar a QV e } \\
\text { o sofrimento } \\
\text { psíquico dos } \\
\text { surdos. }\end{array}$ & $\begin{array}{l}\text { A confiabilidade das } \\
\text { versões do WHOQOL- } \\
\text { Brief e do GHQ-12 } \\
\text { para surdos foi } \\
\text { comparada com a } \\
\text { versão para ouvinte. } \\
\text { Para a BSI, a } \\
\text { confiabilidade foi ainda } \\
\text { maior do que para a } \\
\text { população em geral. }\end{array}$ & $\begin{array}{l}\text { A QV e o sofrimento } \\
\text { mental podem ser } \\
\text { efetivamente avaliados } \\
\text { por instrumentos } \\
\text { traduzidos e adaptados } \\
\text { para LS. }\end{array}$ \\
\hline $\begin{array}{l}\text { Feld Methods/ } \\
\text { Estados Unidos/ } \\
\text { Estados Unidos/ } \\
\text { Inglês/ } 2010\end{array}$ & $\begin{array}{l}\text { A community- } \\
\text { participatory } \\
\text { approach to } \\
\text { adapting } \\
\text { survey items } \\
\text { for deaf } \\
\text { individuals and } \\
\text { American Sign } \\
\text { Language }\end{array}$ & $\begin{array}{l}\text { Tradução para } \\
\text { ASL da } \\
\text { Abordagem } \\
\text { Comunitária } \\
\text { participativa. }\end{array}$ & $\begin{array}{l}\text { Traduzir para ASL } \\
\text { o community- } \\
\text { participatory } \\
\text { approach to } \\
\text { adapting survey } \\
\text { items. }\end{array}$ & $\begin{array}{l}\text { Alcançou-se } \\
\text { equivalência de } \\
\text { significado entre as } \\
\text { línguas envolvidas na } \\
\text { tradução. }\end{array}$ & $\begin{array}{l}\text { Nas investigações com } \\
\text { a população surda, é } \\
\text { imprescindível que os } \\
\text { instrumentos de coleta } \\
\text { de dados sejam em LS. }\end{array}$ \\
\hline
\end{tabular}

ASL (American Sign Language); LS (Língua de Sinais); QV (Qualidade de Vida); WHOQOL (World Health Organization Quality of Life) 
(descritos no Quadro 2) utilizaram instrumentos traduzidos para a língua de sinais, valorizando, assim, a população surda que se constitui como uma minoria linguística e cultural. Dentre os traços culturais da comunidade surda, o que mais se destaca é a língua de sinais; além de ser um sistema linguístico, é um elemento de constituição do sujeito surdo, agregando a identidade e a cultura do povo.

Vários instrumentos de aferição de QVRS, genéricos ou específicos, têm surgido a partir de estudos em diversas culturas. São desenvolvidos em múltiplas línguas, com níveis de equivalência muito mais altos do que jamais se havia feito para uso transcultural. Portanto, destaca-se a necessidade de instrumentos que avaliem a qualidade de vida das pessoas surdas e que eles sejam traduzidos e validados para as diferentes línguas de sinais.

A avaliação da QVRS dos surdos brasileiros que se comunicam pela LIBRAS ainda não foi investigada. As pesquisas encontradas nessa revisão de literatura e que consideram os aspectos linguísticos e culturais da comunidade surda, fazendo a tradução e, alguns, até a validação de instrumentos para a língua de sinais, foram realizadas na Alemanha, na Áustria, na Noruega, na Austrália e nos Estados Unidos.

Usualmente, as medidas de qualidade de vida utilizam questionários para coletar os dados, baseados na língua escrita, excluindo das pesquisas pessoas que não possuem habilidades com a escrita. Os surdos que têm a língua de sinais como primeira língua apresentam dificuldades com a escrita, quando comparados com as pessoas ouvintes. Portanto, utilizar testes escritos para avaliação das pessoas surdas não é apropriado. O baixo nível de leitura das pessoas surdas não interfere, automaticamente, na capacidade de compreensão, se os testes forem apresentados em língua de sinais ${ }^{11}$.

Um estudo com o objetivo de avaliar transtornos mentais e a qualidade de vida de pessoas surdas da Áustria, utilizando os instrumentos WHOQOL-BREF, General Health Questionnaire (GHQ-12) e o Brief Symptom Inventory (BSI), traduzidos e validados para a língua de sinais, verificou que os surdos têm uma qualidade de vida pior do que a da população ouvinte, nos domínios físico e psicológico, medidos pelo WHOQOL-BREF; no entanto, no domínio das relações sociais, não houve diferença significativa. Os resultados com o GHQ-12 e com o BSI mostram níveis mais elevados de problemas emocionais entre os surdos quando comparados com os ouvintes ${ }^{8}$.

Esse resultado pode ser considerado como um indicador de que a convivência na comunidade surda possibilita estabelecer relacionamentos satisfatórios, com base num mesmo sistema de comunicação. Por isso, a língua de sinais tem um papel fundamental para a maioria dos surdos que participam de uma comunidade surda.

Outro estudo² verificou que os DA oralizados tendem a ter uma vida social mais restrita que a das pessoas surdas que participam da cultura surda e usam a língua de sinais. Os DA são excluídos pelas pessoas ouvintes por causa da sua deficiência, e eles precisam encontrar, constantemente, meios para sobreviver na cultura dos ouvintes. Os surdos que usam a língua de sinais e participam da comunidade surda podem conseguir bons desempenhos em suas relações sociais, mas os DA podem apresentar uma vida social restrita.

Ao encontrar pacientes que têm dificuldades de comunicação, como os surdos, os profissionais devem estar cientes do grande impacto que tais dificuldades têm sobre a qualidade de vida e o estresse emocional $^{3}$. Dificuldades de comunicação no dia a dia das pessoas surdas é uma condição permanente, por fazerem parte de um grupo minoritário que não se comunica pela língua oral do seu país. Além disso, é uma experiência frustrante e comum para os surdos que vivem numa família ouvinte. Essa barreira de comunicação pode provocar, como uma das consequências, problemas emocionais e de conduta, podendo justificar os altos níveis de transtornos mentais relatados em pesquisa $2,8,12$.

Em relação aos ouvintes, os surdos que se comunicam pela língua de sinais mostram níveis de estresse psicológico mais elevados, todavia, nas relações sociais não apresentam diferença significativa. Já os DA oralizados apresentaram mais problemas em suas relações sociais, e em todos os escores avaliados estão em desvantagem em relação à população ouvinte e surda².

Na comparação entre surdos e ouvintes, verifica-se que os sintomas de ansiedade e depressão são maiores nos grupos de surdos. Destacamos isso como um indicativo de que a sociedade deve despender maior esforço para diminuir as barreiras que estão associadas à surdez. Pessoas surdas, independentemente da idade, devem ter garantida a sua acessibilidade comunicacional na sociedade. 


\section{Artigos que investigaram a qualidade de vida com instrumentos sem tradução ou com tradução simultânea para a língua de sinais}

Nos últimos anos, o aspecto subjetivo da qualidade de vida das pessoas influenciou as deliberações políticas e científicas em saúde. O foco na qualidade de vida é importante para todos, mas assume um significado especial para pessoas que vivem na sociedade em condições mais difíceis do que as de outras pessoas. Particularmente no caso dos surdos, é necessário conhecer suas necessidades como parte integrante da sociedade e quais as condições para realizar seus valores subjetivos ${ }^{13}$.

Uma pesquisa ${ }^{14}$, com uma amostra composta por crianças surdas, seus pais e professores, identificou, nas respostas dos pais e professores, que as crianças surdas apresentam mais problemas de qualidade de vida do que crianças ouvintes. Essas diferenças estão mais relacionadas a problemas de conduta, emocionais e de relacionamento (por exemplo, isolamento social), indicando como possível etiologia destes problemas a dificuldade de uma comunicação efetiva. Nesse mesmo estudo, não houve diferença significativa em saúde mental com relação ao grau da perda auditiva.

As pessoas que vivenciam problemas de comunicação evitam novas relações sociais, conduzindo-se ao isolamento social e, consequentemente, à redução da sua qualidade de vida. A satisfação com a vida e a autoestima são indicadores essenciais para a qualidade de vida e a saúde mental dos surdos e dos DA. Em contrapartida, uma baixa autoestima e a não-aceitação da perda auditiva são importantes desencadeadores de transtornos mentais. O estudo também revelou que a autoestima foi maior entre os surdos cujos pais utilizavam a língua de sinais em casa, quando comparados com aqueles cujos pais preferiam uma educação oral ${ }^{7,14}$.

O primeiro relato, com o objetivo de investigar os aspectos da qualidade de vida de surdos idosos com perda auditiva pré-lingual, usuários da língua de sinais, com foco no bem-estar, na depressão e na insônia, demonstrou que sintomas depressivos e distúrbios do sono são mais frequentes entre a população surda que usa a língua de sinais do que entre as pessoas ouvintes. No entanto, ao contrário das expectativas dos autores, a comparação do bem-estar não apresentou respostas inferiores em relação à população idosa ouvinte ${ }^{15}$.

A prevalência atual de grande parte das pesquisas sobre o desenvolvimento psicossocial mostra que a frequência de alterações socioemocionais é muito maior em crianças surdas e em crianças DA do que em crianças ouvintes, com estimativa de um aumento de 2,6 para 3,6 vezes em relação à amostra de ouvintes. Os resultados desses estudos psicossociais mostram que a qualidade de vida dos surdos e DA pode estar em risco, e esse tema merece mais atenção dos pesquisadores ${ }^{13}$.

Outro estudo ${ }^{14}$ revelou evidências de que a qualidade de vida percebida pela criança surda e pela criança DA não foi relacionada à situação auditiva (grau da perda e implante coclear). Isso demonstra que a satisfação com a vida e a autoconfiança não são determinadas pelos graus da perda auditiva ${ }^{13,16}$. Fica evidente que uma forte integração social e cultural e condições de alcançá-la são mais relevantes do que o nível de acuidade auditiva.

No entanto, resultados diferentes foram apresentados em outra pesquisa ${ }^{17}$, com relação à influência da perda auditiva na QVRS. De modo geral, os indivíduos com perda auditiva profunda relataram menor QVRS. Diferenças significativas foram obtidas por falta de energia, reações emocionais e isolamento social. Nesse mesmo estudo, verificou-se que as mulheres apresentaram QVRS mais baixa que a dos homens. As pessoas com deficiência auditiva profunda constituem um grupo de risco, com pior ajustamento psicossocial, e precisam de maior atenção e apoio.

\section{Artigos que fizeram a tradução de instrumentos para as línguas de sinais}

Instrumentos padronizados são fundamentais para fazer avaliações em saúde e para mensurar o impacto dos planejamentos das intervenções públicas em saúde, com possibilidade de reduzir as desigualdades. No entanto, o uso de instrumentos padronizados que não tenham sido previamente testados e validados para aplicação na população surda, aumenta o risco de se chegar a conclusões inadequadas nas investigações clínicas. Por isso, instrumentos desenvolvidos para aplicação na comunidade surda precisam ser confiáveis, com o rigor de uma validação, incluindo as especificidades 
da cultura surda. Praticamente não há instrumentos de avaliação da QVRS e da saúde mental padronizados e validados para uso na população surda que se comunica pela língua de sinais ${ }^{18}$.

Sem um desfecho válido e confiável em Língua de Sinais Australiana, dos instrumentos "Outcome Rating Scale" (ORS) e "Depression Anxiety Stress Scale-21" (DASS-21), pesquisadores realizaram a tradução e a validação desses instrumentos, com a finalidade de assegurar a eficácia das intervenções em saúde mental para usuários de língua de sinais. O objetivo do estudo foi examinar a confiabilidade, a validade e a aceitabilidade da versão Língua de Sinais Australiana do ORS e do DASS- $21^{19}$.

Os resultados desse estudo ${ }^{19}$ indicaram diferenças significativas entre as médias para a amostra clínica e as da comunidade surda. A consistência interna foi aceitável, dado o baixo número de itens na ORS-Australiana. A validade de construto foi estabelecida pela correlação significativa entre a pontuação total da DASS-21-Australiana e da ORS-Australiana. A aceitabilidade da ORS-Australiana ficou evidente na taxa de conclusão de $93 \%$ em comparação com $63 \%$ para a DASS-21-Australiana. Com um desfecho disponível na versão em Língua de Sinais Australiana, os profissionais têm à sua disposição um instrumento que pode ser usado nas avaliações de saúde mental e clínica da população surda.

Um estudo ${ }^{18}$ apresentou a metodologia de tradução do Multidimensional Health Locus of Control (MHLC) em escalas na American Sign Language (ASL). O MHLC foi traduzido utilizando grupos focais, compostos por cinco participantes bilíngues que traduziram a MHLC em ASL, e outros cinco que retrotraduziram a versão ASL para o inglês. Os grupos focais identificavam e corrigiam os problemas de linguagem e culturais antes da versão final do MHLC em ASL. Nove dos 24 itens foram diretamente traduzíveis em ASL. Os demais itens necessitaram de mais discussões para alcançar equivalência com as expressões culturais em ASL. O MHLC/ASL foi validado no âmbito da comunidade surda. Todo processo de tradução foi feito em Digital Versatile Disk (DVD), o que possibilitou a tradução e a validação na comunidade surda.

Os instrumentos para serem usados com confiança em diversas comunidades devem, primeiro, ser traduzidos nas línguas dessas comunidades. O padrão ouro para o processo de tradução deve apresentar:

- grupo focal bilíngue e bicultural;

. primeiramente, as traduções e, posteriormente, a retrotradução;

. reconciliação dos itens com conceitos divergentes, buscando a equivalência funcional;

. instrumentos de tradução testados, para determinar se eles são confiáveis e válidos para aplicação nas comunidades;

. normas estabelecidas por meio de práticas de pesquisa ${ }^{18}$.

Traduzir um instrumento para língua de sinais é particularmente difícil, mas essencial. As dificuldades são, sobretudo, em relação à modalidade de produção da língua de sinais, que é espaço-visual, pois a versão impressa dessa língua ainda não é acessível a todos os surdos. Assim, torna-se necessário recorrer a alternativas, como apresentação em vídeo. Qualquer instrumento em língua oral, se for aplicado em uma comunidade surda, não oferece uma avaliação confiável, sendo, portanto, inadequada a utilização de instrumentos em línguas orais para os membros da comunidade surda que têm a língua de sinais como língua natural ${ }^{18,20}$.

A comunidade surda afirma que somente com instrumentos em língua de sinais é possível atingir os objetivos que um estudo se propõe, pois, com instrumentos adequados, o significado implícito das questões é compreendido pelos surdos que irão responder ao questionário. Isso é reforçado na literatura que defende a aplicação de instrumentos na primeira língua da comunidade; quando o instrumento está em uma segunda língua, os resultados são questionáveis ${ }^{18,21}$.

Um grupo de pesquisadore ${ }^{11}$ estruturou uma avaliação de qualidade de vida e de saúde mental para pessoas surdas, os instrumentos WHOQOL-BREF, General Health Questionnaire (GHQ - 12 itens) e Brief Symptom Inventory (BIS) foram traduzidos para a língua de sinais. Quando a confiabilidade das versões do WHOQOL-BREF e o GHQ-12 para a língua de sinais foi comparada com a versão escrita nas mesmas medidas em amostras da população ouvinte, constatou-se ser um pouco menor, embora ainda em um intervalo aceitável, para a OMS. Para a BSI, o teste de confiabilidade foi ainda maior do que o da população em geral. Esse trabalho desenvolveu as versões em língua de sinais dos instrumentos WHOQOL-BREF, GHQ - 12 itens e BIS, em um programa de computador. Essa versão é 
autoadministrada e consiste em questões em língua de sinais gravadas em vídeo, com apresentação simultânea da língua oral escrita. Para surdos que se comunicam pela língua de sinais, a qualidade de vida e a saúde mental podem ser efetivamente avaliadas por instrumentos traduzidos e adaptados para essa população.

Outro estudo ${ }^{20}$ propôs uma tradução e adaptação dos itens da Abordagem Comunitária Participativa para surdos que se comunicam em língua de sinais. Por meio da retrotradução, foi possível alcançar o significado de equivalência entre as línguas envolvidas na tradução. Duas amostras diferentes de surdos adultos (302 e 215 surdos) responderam ao questionário. As análises indicam que mais de 75\% dos itens da pesquisa foram respondidos em padrões comparáveis nas duas amostras.

Quando os instrumentos de pesquisa refletem os valores da cultura dominante que não são partilhados pela cultura minoritária, os dados podem estar comprometidos. Um projeto de pesquisa transcultural contém desafios complexos no estilo, na tradução, na amostragem e outros; e ainda deve atender aos princípios éticos e, no caso dos surdos, incluir os aspectos específicos da população surda.

\section{Conclusão}

Um dos desafios para a sociedade, no século XXI, se traduz no respeito à diversidade e no direito à igualdade. Para tanto, deve-se incluir a convivência com pessoas com deficiência. Nas relações sociais, essas pessoas encontram barreiras além daquelas experimentadas por outras sem deficiência. Esse peso extra pode aumentar o risco de as pessoas com deficiência desenvolverem problemas de saúde mental, o que pode reduzir a sua qualidade de vida.

A revisão de literatura demonstrou que a menor qualidade de vida das pessoas surdas é esperada pela dificuldade de comunicação, o que pode gerar uma vulnerabilidade dos surdos aos problemas de saúde mental. Os sintomas de ansiedade e depressão são maiores nos surdos, quando comparados com a população ouvinte. As pessoas que vivenciam problemas de comunicação evitam novas relações sociais, e isso, a longo prazo, pode aumentar o isolamento social e reduzir a sua qualidade de vida.

Para os surdos que se comunicam pela língua de sinais, a qualidade de vida só pode ser efetivamente avaliada por instrumentos traduzidos e adaptados para essa população. No entanto, os instrumentos de avaliação na área da saúde têm sido desenvolvidos e testados em línguas orais e com pessoas ouvintes. Por isso, usuários da língua de sinais, quando respondem aos instrumentos baseados em línguas orais, encontram dificuldades, pois eles não contemplam os aspectos culturais e linguísticos da comunidade surda.

Diante disso, destaca-se a necessidade de instrumentos validados, com versão em Libras, que avaliem a QVRS das pessoas surdas brasileiras. Como existem, em língua de sinais, poucos registros de instrumentos na área da saúde, e menos ainda de avaliação da qualidade de vida, é urgente pensar em instrumentos em Libras para avaliar a QVRS da população surda do Brasil.

\section{Colaboradores}

Os autores Neuma Chaveiro, Soraya Bianca Reis Duarte, Adriana Ribeiro de Freitas, Maria Alves Barbosa, Celmo Celeno Porto, Marcelo Pio de Almeida Fleck participaram, igualmente, de todas as etapas de elaboração do artigo. 


\section{Referências}

1. Sacks O. Vendo vozes: uma viagem ao mundo dos surdos. Trad. de Laura Teixeira Motta. São Paulo: Companhia das Letras; 1998.

2. Fellinger J, Holzinger D, Gerich J, Goldberg D. Mental distress and quality of life in the hard of hearing. Acta Psychiatr Scand. 2007; 11:243-45.

3. Chaveiro N, Barbosa MA, Porto CC, Munari DB, Medeiros M, Duarte SBR. Atendimento à pessoa surda que utiliza a língua de sinais, na perspectiva do profissional da saúde. Cogitare Enferm. 2010; 15(4):639-45.

4. Decreto n $\mathrm{n}^{\circ}$ 5.626, de 22 de dezembro de 2005. Regulamenta a Lei $\mathrm{n}^{\circ} 10.436$, de 24 de abril de 2002, que dispõe sobre a Língua Brasileira de Sinais - Libras e o art. 18 da Lei no 10.098, de 19 de dezembro de 2000. Diário Oficial da República Federativa do Brasil. 2005 Dez 23.

5. Chaveiro N, Barbosa MA, Porto CC. Literature revision about the attendance of deaf patient by health professionals. Rev Esc Enferm USP. 2008; 42(3):578-83.

6. Oliveira YCA, Costa GMC, Coura AS, Cartaxo RO, França ISX. A língua brasileira de sinais na formação dos profissionais de enfermagem, fisioterapia e odontologia no estado da Paraíba, Brasil. Interface (Botucatu). 2012; 16(43):995-1008.

7. Graaf RD, Bilj RV. Determinants of mental distress in adults with a severe auditory impairment: differences between prelingual and postlingual deafness. Psychosom. Med. 2002; 64:61-70.

8. Fellinger J, Holzinger D, Dobner U, Gerich J, Lehner R, Lenz G, et al. Mental distress and quality of life in a deaf population. Soc Psychiatry Psychiatr Epidemiol. 2005; 40(9): 737-42.

9. Mendes KDS, Silveira RCCP, Galvão CM. Revisão integrativa: método de pesquisa para incorporação de evidências na saúde e na enfermagem. Texto Contexto Enferm. 2008; 17(4):758-64.

10. Fleck MPA, Lousada S, Xavier M, Chachamovich E, Vieira G, Santos L, et al. Desenvolvimento da versão em português do instrumento de avaliação de qualidade de vida da OMS (WHOQOL-100). Rev Bras Psiquiatr. 1999; 21(1):19-28.

11. Fellinger J, Holzinger D, Dobner U, Gerich J, Lehner R, Lenz G, et al. An innovative and reliable way of measuring health-related quality of life and mental distress in the deaf community. Soc Psychiatry Psychiatr Epidemiol. 2005; 40(3):245-50.

12. Kvam MH, Leob M, Tambs K. Mental health in deaf adults: symptoms of anxiety and depression among hearing and deaf individuals. J Deaf Stud Deaf Educ. 2007; 12(1):1-7.

13. Hintermair M. Self-esteem and satisfaction with life of deaf and hard-of-hearing people - a resource-oriented approach to identity work. J Deaf Stud Deaf Educ. 2008; $13(2): 278-300$.

14. Fellinger J, Holzinger D, Sattel H, Laucht $M$. Mental health and quality of life in deaf pupils. Eur Child Adolesc Psychiatry. 2008; 17:414-23.

15. Werngren-Elgström M, Dehlin O, Iwarsson S. Aspects of quality of life in persons with pre-lingual deafness using sign language: subjective wellbeing, ill-health symptoms, depression and insomnia. Arch Gerontol Geriatr. 2003; 37(1):13-24.

16. Hintermair $M$. Health-related quality of life and classroom participation of deaf and hard-of-hearing students in Genera schools. J Deaf Stud Deaf Educ. 2010; 16(2):254-71.

17. Ringdahl A, Grimby A. Severe-profound hearing impairment and health-related quality of life among post-lingual deafened Swedish adults. Scand Audiol. 2000; 29(4):266-75 
18. Samady W, Sadler GR, Nakaji M, Malcarne VL, Trybus R, et al. Translation of the multidimensional health locus of control scales for users of American sign language. Public Health Nurs. 2008; 25(5):480-9.

19. Munro L, Rodwell J. Validation of an Australian sign language instrument of outcome measurement for adults in mental health settings. Aust. N Z J Psychiatry. 2009; 43(4):332-9

20. Graybill P, Aggas J, Dean RK, Demers S, Finigan EG, Jr. Pollard RQ. A communityparticipatory approach to adapting survey items for deaf individuals and American Sign Language. Feld Methods. 2010; 22(4):429-48.

21. Jones EG, Mallinson RK, Phillips L, Kang Y. Challenges in language, culture, and modality: translating English measures into American Sign Language. Nurs Res. 2006; 55(2):75-81

Chaveiro N, Duarte SBR, Freitas AR, Barbosa MA, Porto CC, Fleck MPA. Calidad de vida de los sordos que se comunican por la lengua de signos: revisión de integración. Interface (Botucatu). 2014; 18(48):101-14.

El objetivo del estudio fue revisar la producción científica sobre la Calidad de Vida Relacionada con la Salud (QVRS, por sus siglas en portugués) de los sordos. Se trata de una revisión de integración realizada en la Biblioteca Virtual en Salud, PubMed y Portal de periódicos de la Coordinación de Perfeccionamiento de Personal de Nivel Superior (Capes). Los resultados indican que los síntomas de ansiedad y depresión son más acentuados en los sordos y pueden estar relacionados con dificultades de comunicación. Las personas que tienen problemas de comunicación evitan nuevas relaciones sociales y eso puede aumentar el aislamiento social y reducir la QVRS. Para los sordos que se comunican por la Lengua de Signos, la QVRS solamente puede evaluarse efectivamente por medio de instrumentos traducidos y adaptados a su lengua. Se concluye que la sordera causa un impacto negativo sobre la QVRS de los sordos.

Palabras-clave: Sordera. Lengua de signos. Calidad de vida. Revisión. 